\section{Evaluación de las proporciones de almidón de yuca y emulsificantes para el mejoramiento textural de una torta libre de gluten}

\section{Mariana Vélez Gutierrez* Laura Cristina Jimenez, Ximena Yepes Sánchez}

Universidad de Antioquia, Facultad de Ciencias Farmacéuticas y Alimentarias, Ingeniería de Alimentos, Medellín, Colombia

* Autor para correspondencia / correo electrónico: marianavelez9@gmail.com

\section{Evaluation of proportions of cassava starch and emulsifiers for textural improvement of gluten free cake}

\section{Abstract}

Celiac disease is an autoimmune enteropathy precipitated by exposure to gluten in foods such as wheat, barley and rye in individuals who are genetically predisposed. In the last ten years the production of gluten-free foods at the global level has increased almost 28\%, including traditional products in which it seeks to ensure that these comply with physical and sensory characteristics inherent in the product. The objective of this work was to perform a design of experiments in response surface to evaluate the different proportions of starch from cassava and emulsifiers in the elaboration of a gluten free cake and thus improve textural attributes. Eleven were developed cakes varying only the percentage of starch from cassava and emulsifiers as obtained in the design of experiments; to each one of them were determined the pore size and height in triplicate, and specific gravity by means of the AACC 10-90. The response variable obtained were optimized in the Software Design Expert 8.0 giving as a result a Severity Specifies minimized of $0.8014 \%$, maximized height of $65.711 \mathrm{~mm}$ and a range of pore size of between $1.9-2.5 \mathrm{~mm}$, it was subsequently determined the optimal point with the amount of emulsifier and cassava starch suitable for the elaboration of a gluten free product with the desired textural features (emulsifier 1.5\% and starch from cassava 19.65\%). Emulsifiers and cassava starch in appropriate proportions allow for physical and sensory characteristics similar to those which gives the wheat flour and can be consumed by people who suffer from celiac disease as an alternative supplement to your daily diet.

Key words: Cake, gluten-free flours, emulsifiers, cassava starch.

\section{Resumen}

La enfermedad celíaca es una enteropatía autoinmune precipitada por la exposición al gluten en alimentos como el trigo, la cebada y el centeno en individuos genéticamente predispuestos. En los últimos 10 años la producción de alimentos libres de gluten a nivel mundial ha aumentado casi un $28 \%$, incluyendo productos tradicionales en los que se busca que estos cumplan con características físicas y sensoriales propias del producto. El objetivo de este trabajo fue realizar un diseño de experimentos de superficie de respuesta para evaluar las diferentes proporciones de almidón de yuca y emulsificantes en la elaboración de una torta libre de gluten y así mejorar atributos texturales. Se elaboraron once tortas variando únicamente el porcentaje de almidón de yuca y emulsificantes según lo obtenido en el diseño de experimentos; a cada una de ellas se les determinó el tamaño del poro y altura por triplicado y gravedad espeć́fica por medio de la AACC 10-90. Las variables respuesta obtenidas fueron optimizadas en el software Desing Expert 8.0 dando como resultado una gravedad específica minimizada de $0.8014 \%$, la altura maximizada de $65.711 \mathrm{~mm}$ y un rango de tamaño de poro entre 1.9-2.5mm, posteriormente se determinó el punto óptimo con la cantidad de emulsificante y almidón de yuca adecuados para la elaboración de un producto libre de gluten con las características texturales deseadas (emulsificante 1,5\% y almidón de yuca 19,65\%). Los emulsificantes y el almidón de yuca en proporciones adecuadas permiten obtener características físicas y sensoriales similares a las que otorga la harina de trigo y puede ser consumido por personas que padecen de celiaquía como un suplemento alternativo a su dieta diaria.

Palabras claves: Torta, Harinas libres de gluten, Emulsificantes, Almidón de yuca

\section{INTRODUCCIÓN}

a enfermedad celíaca (EC) es una enteropatía crónica autoinmune precipitada, en individuos genéticamente predispuestos, por la ingestión de gluten. El gluten es un conjunto de proteínas de almacenamiento presentes en diferentes cereales, incluyendo el trigo, el centeno, la cebada y la avena (las aveninas de la avena son consideradas como un tipo de gluten, pero su nivel de inmunoreacción va a depender del cultivar consumido; además, la presencia de gluten en avena puede aparecer por contaminación cruzada) [1-3]. La EC conlleva un aumento del riesgo de varias enfermedades malignas, incluyendo los cánceres y los tumores malignos hematológicos del tracto gastrointestinal [4]. El único tratamiento efectivo para la EC es adherirse a una dieta libre de gluten. En 2013, la Administración de Medicamentos y Alimentos de los Estados Unidos (FDA) emitió una regla final para la definición de "libre de gluten"en el etiquetado de alimentos [5]. Sin embargo, actualmente las tendencias del mercado no favorecen a las personas intolerantes al gluten. A pesar de que en los últimos 10 años la producción de alimentos libres de gluten ha aumentado casi un $28 \%$ a nivel mundial, la ingesta de alimentos es limitada debido a la poca variedad de productos que ofrece el mercado y su alto costo [6]., Casi la mitad de los pacientes que tienen EC consideran que llevar una dieta libre de gluten implica un costo mucho más alto que el costo de una dieta regular [7]. Por lo tanto, es importante explorar nuevas opciones que ayuden a extender la dieta diaria pensando en las personas que padecen EC y buscando ampliar el portafolio de alternativas para la población en general. Esto incluye el desarrollo de productos tradicionales que no pongan en riesgo la salud, que cumplan con características fisicoquímicas y sensoriales aceptables y propias del producto tradicional con gluten, y que sean de bajo costo. Desde la ingeniería de alimentos es posible subsanar el vacío a nivel de alimentos libres 

12 de gluten en Colombia. La opción a futuro es encontrar opciones libres de gluten, para de vida saludables.

El uso del gluten se masificó debido a su capacidad de retener aire en la matriz proteica facilitando que la masa se adhiera mejor, fenómeno que favorece la elaboración de pan. La producción de alimentos horneados sin adición de harina de trigo representa un importante reto tecnológico, debido a la ausencia de gluten que genera las un importante reto tecnológico, debido a la ausencia de gluten que genera las
propiedades visco-elásticas. Para compensar, algunos hidrocoloides se incorporan menudo en formulaciones libres de gluten [8]. Estos hidrocoloides actúan como agentes emulsionantes y como agentes de estabilización emulsionante y su función principal es unir moléculas de agua y aceite de forma homogénea, logrando un balance hidrolipofilico. Es así, que estos emulsificantes son acondicionadores de masa y ablandadores de la miga [8]. Los emulsificantes mejoran las propiedades físicas de los productos, proporcionando mayor volumen por la captación de aire durante el amasado. También, se observa migas más flexibles, con alveolados más finos y uniformes, y como consecuencia de una mayo tolerancia se reducen los problemas derivados de la fatiga de la masa en su transformación por las máquinas. Los mono-y diglicéridos de los ácidos grasos son muy utilizados como emulsionantes en la elaboración de batidos de pastelería [9]. Los emulsionantes tienen la posibilidad de sustituir parcialmente la grasa, el azúcar o ambos en una formulación pastelera. El uso de emulsionantes provee mayor expansión global, mejor distribución de los alvéolos de la miga y homogeneidad de éstos y se puede mejorar el perfil de textura durante el almacenamiento del producto [10]

El almidón es el carbohidrato de mayor abundancia en la naturaleza. Su influencia es importante ya que cuanto mayor es su contenido, el producto resulta más adhesivo, característica que se aprovecha extensamente como agente espesante, estabilizante y adhesivo en la industria alimentaria [11]. El almidón de yuca posee características y adhesivo en la industria alimentaria [11]. El almidón de yuca posee caracteristicas
para mejorar la textura, impartir viscosidad, ligar agua, proveer cohesión y mantener la tolerancia a los procesos, necesaria y requerida para la manufactura. Estos almidones tienen la propiedad de prolongar la vida útil de los alimentos garantizando a su vez calidad [12].

La finalidad de esta investigación fue la elaboración de una torta de naranja libre de gluten con base en una formulación estándar con harina de maíz y harina de arroz, para sustituir la harina de trigo, y la adición de almidón de yuca y emulsificantes en diferentes concentraciones para encontrar el punto óptimo textural adecuado.

\section{MATERIALES Y MÉTODOS}

Las mezclas de tortas compuestas por harina de trigo, huevos, grasa y azúcar, exhiben comportamiento viscoelástico, combinando las propiedades de un fluido y un sólido. Cada ingrediente es esencial en la mezcla, exhibiendo un rol importante en la definición estructural, reológica y textural de las propiedades de la torta. Cualquier cambio realizado en los ingredientes básicos o proporciones de los mismos conlleva a un cambio en las propiedades físicas, químicas y sensoriales del producto final [13]. El proceso de elaboración de una torta implica modificaciones estructurales dependiendo de la formulación y las condiciones del proceso, las cuales son fenómenos de importancia para la calidad de la torta y pueden ser investigadas por medio de análisis reológicos y estructurales, usando técnicas como la medición de la viscosidad y análisis de imágenes correlacionando las concentraciones de gluten con los cambios generados en las propiedades de la mezcla de la torta durante el horneado y con el producto final [14]. Las propiedades texturales de un producto juegan un papel muy importante en la apreciación de los alimentos; ya que surge de la interacción al momento de consumirse. Esta percepción constituye un criterio para controlar la calidad y es un factor fundamental de selección o rechazo. Al mezclar batidos, por ejemplo, es importante medir la cantidad de aire incorporado a la mezcla, lo cual se determina mediante la medición de la gravedad específica [15]. Un rango intermedio para este parámetro (0.5 a 0.6) es usado para tortas esponjosas como el bizcochuelo, mientras que un rango alto (0.9 a 0.95) es común para tortas pesadas como los 'layer cakes' [16].

\section{Materia Prima}

Se utilizó harina de arroz y harina de maíz suministrada por la empresa C.I. Atussa Ltda (Itagüí, Antioquia, Colombia); con las siguientes características: humedad, 10-13,5\%; grasa, $0,5-1,5 \%$; fibra, $0,1 \%$; cenizas, 0,2-0,4\%; proteína, 5,8\%; carbohidratos totales, 80,7\%; calorías, 352,3 Kcal/100g; índice de gelatinización, 21,72. Las dos harinas estuvieron libres de gluten. Para la preparación de la torta se usó una proporción de harinas de 1:1. La goma Xantan fue obtenida por medio de la empresa Bell Chem Internacional S.A.,

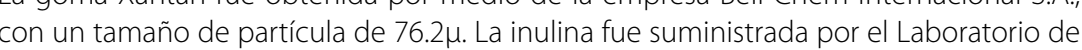
Lácteos de la Universidad de Antioquia, siendo el proveedor directo Tecnas S.A., con un grado de pureza de $\geq 92 \%$. El almidón de yuca fue provisto por la empresa Colorisa S.A. Los estabilizantes usados fueron monoestearato de glicerilo y polisorbato 60 suministrados por la empresa Bell Chem Internacional S.A. Los siguientes ingredientes, utilizados en la evaluación de las tortas, fueron adquiridos en un mercado local de la ciudad de Antioquia (marca comercial o variedad entre paréntesis): harina de trigo (Haz de Oros), huevos (Avinal), azúcar (Manuelita), polvo de hornear (Condimentos El Rey), sal (Refisal), margarina (La Fina), y naranjas (Navelate).

\section{Preparación de la Torta}

La formulación inicial de la torta fue obtenida por medio de porcentajes panaderos, es decir, la cantidad total de harinas de la mezcla (harinas de arroz y de maíz) se tomó como el 100\% y a partir de ahí se calcularon los porcentajes adecuados para cada uno de los ingredientes. El proceso de batido y formulación de la torta fueron estandarizados bajo condiciones, tiempos, temperaturas y equipos constantes con el fin de tener la menor variabilidad en las mediciones. El batido se realizó con ayuda de una batidora de mano Black\&Decker ${ }^{\oplus}$ Spatula Smart ${ }^{\oplus}$ de 3 velocidades. Inicialmente se realizó una mezcla y homogeneización completa del azúcar con la mantequilla en velocidad 3 durante 3.31 minutos (3000rpm), luego se añadió los ingredientes sólidos (previamente mezclados) y líquidos alternadamente para lograr una total homogeneización de la mezcla. Este proceso se realizó durante 7.22 minutos en velocidad 3 para generar la máxima incorporación de aire en el batido. Se utilizó moldes circulares estándar en los cuales se horneó individualmente $400 \mathrm{~g}$ de mezcla para cada formulación. El horneo 
se llevó a cabo en un horno de convección Salva KWIK-CO que logra mantener una temperatura uniforme en su interior, gracias a sus ventiladores internos. Se manejó un tiempo estándar de 32 minutos de horneo a $140^{\circ} \mathrm{C}$ para cada torta. Este tipo de horno ahorra un 20\% de tiempo de preparación a temperaturas más bajas, a diferencia de los hornos convencionales. Cada formulación fue desarrollada independientemente, es decir, cada formulación tuvo un proceso independiente desde la preparación de la premezcla hasta el horneo para evitar desviaciones en el diseño estadístico. Las tortas fueron retiradas del horno y desmoldadas 10 minutos después, posteriormente se rueron retiradas del horno

\section{Medición de la gravedad específica de la masa}

Con el fin de conocer la cantidad de aire incorporado a la mezcla, se realizó la medición de la gravedad específica cuyo resultado adimensional numéricamente coincide con la densidad. Para la medición se tomó un recipiente de $8,7 \mathrm{~cm}$ de diámetro y $4 \mathrm{~cm}$ de alto se lo pesó completamente lleno de agua y posteriormente se vertió dentro el batido, evitando la presencia de burbujas grandes y emparejando el recipiente con una espátula. La medición de la gravedad específica se hizo una sola vez y se calculó de acuerdo a la siguiente ecuación (1):

$$
\mathrm{GE}=\frac{\text { Peso del Batido }(\mathrm{g})}{\text { Peso del } \mathrm{agua}(\mathrm{g})}
$$

\section{Medición del tamaño de poro y altura de la torta}

La medición de las variables tamaño de poro y altura de la torta fueron realizadas utilizando el software Image J, un programa de procesamiento digital de imágenes que permite tomar una escala de referencia y medir distancias sobre una imagen determinada con apreciación subjetiva de las imágenes. Se tomó tres fotografías a cada tipo de torta directamente sobre su vista transversal. En total se obtuvo 33 imágenes durante los experimentos que fueron analizadas realizando mediciones por triplicado de las variables del tamaño del poro y altura, utilizando siempre el mismo operador para mantener constantes los errores sistemáticos. La medición de tamaño de poro se realizó escogiendo un poro de la torta de cada fotografía, después de tener la escala establecida en centímetros se traza una línea horizontal en medio del poro y el software arroja la medida exacta de este trazo que corresponde al diámetro del poro. Para la medición de altura, después de tener la escala establecida en centímetros, se toma un punto en la parte inferior y central de la torta en cada fotografía y allí se traza una línea hasta la corteza de la torta, el software arroja la medida de esta línea que corresponde a la altura de la torta. Estas medidas se reportan con 3 cifras significativas.

Diseño Experimental
Se realizó una optimización del proceso de elaboración de la torta libre de gluten mediante un diseño de superficie de respuesta IV Optimal, en función de dos factores en la formulación: porcentaje del emulsificante (0-2\%) y porcentaje del almidón de yuca (0-50\%), generando 11 unidades experimentales utilizando el software Desing Expert 8.0. Las variables dependientes a optimizar fueron: gravedad específica, tamaño de poro y altura de la torta (Tabla 1). El análisis estadístico se realizó mediante análisis de varianza (ANOVA), donde las diferencias significativas y las correlaciones se llevaron a cabo en los datos experimentales adoptando el método de diferencia mínima significativa con un nivel de significancia menor o igual a un $5 \%$. El análisis de regresión para los modelos de cada variable respuesta en función de los factores de diseño fue de la forma:

\section{$Y=\beta_{0}+\beta_{1} X_{1}+\beta_{2} X_{2}+\beta_{12} X_{1} X$}

Donde es la variable respuesta, y son los factores, es el intercepto, $\beta_{11} \beta_{2}$ y $\beta_{12}$ son los efectos asociados a cada factor y su interacción respectivamente

Tabla 1. Diseño experimental IV Optimal de superficie de respuesta para tortas libres de gluten con emulsificantes y almidón de yuca en diferentes concentraciones.

\begin{tabular}{|cccc|}
\hline Aleatorización & Corrida & Emulsificante (\%) & Almidón Yuca (\%) \\
\hline 9 & 1 & 0,00 & 50,00 \\
\hline 1 & 2 & 0,00 & 0,00 \\
\hline 6 & 3 & 1,79 & 25,00 \\
\hline 2 & 4 & 2,00 & 0,00 \\
\hline 11 & 5 & 2,00 & 50,00 \\
\hline 8 & 6 & 0,00 & 50,00 \\
\hline 7 & 7 & 1,00 & 42,50 \\
\hline 3 & 8 & 2,00 & 0,00 \\
\hline 10 & 9 & 2,00 & 50,00 \\
\hline 5 & 10 & 0,21 & 25,00 \\
\hline 4 & 11 & 1,00 & 7,50 \\
\hline
\end{tabular}

Finalmente, con los resultados obtenidos del punto óptimo se realizó una comparación múltiple por medio del Test de Fisher entre las medias de cada uno de los factores respecto al modelo calculado.

\section{RESULTADOS}

Se elaboró once tortas bajo las mismas condiciones de temperatura y tiempo controladas. La gravedad específica varió entre 0,801 y 1,068, el tamaño de poro entre 1,801 y 2,798mm y la altura de la torta entre 25.246 y $65.712 \mathrm{~mm}$ (Tabla 2), dependiendo de cada corrida.

Tabla 2. Gravedad específica, tamaño de poro y altura de tortas libres de gluten con emulsificantes y almidón de 
yuca en diferentes concentraciones.

\begin{tabular}{cccc} 
Corrida & Gravedad especifica & Tamaño de poro $(\mathrm{mm})$ & Altura torta $(\mathrm{mm})$ \\
\hline 1 & 0,947 & 2,413 & 25,246 \\
2 & 1,068 & 1,801 & 35,125 \\
3 & 0,801 & 2,463 & 61,522 \\
4 & 0,845 & 2,527 & 65,712 \\
5 & 0,890 & 2,768 & 29,049 \\
6 & 0,940 & 2,548 & 27,415 \\
7 & 0,891 & 2,798 & 40,589 \\
8 & 0,825 & 2,557 & 63,830 \\
9 & 0,880 & 2,684 & 32,795 \\
10 & 0,880 & 2,235 & 52,911 \\
11 & 0,960 & 2,165 & 45,842
\end{tabular}

La cantidad de emulsificantes, la adición de almidón de yuca y su interacción influyeron significativamente en el tamaño de poro entre cada una de las corridas (Tabla 3, Fig. 1). La medida de la gravedad específica establece la cantidad de aire que se ha incorporado a la mezcla durante el batido en relación a la densidad del agua. Valores mayores a 1,0 son característicos de batidos pesados y que durante el horneo no tendrán la capacidad de crecer, por el contrario, valores por debajo indican que la mezcla es más liviana que el agua y ha incorporado una cantidad de aire que le permitirá desarrollar mejores características sensoriales en el producto final. Como se esperaba la adición de emulsificantes tuvo una gran significancia en la gravedad específica del batido, el porcentaje de almidón por si solo también fue significativo en la medición de esta variable, pero en menor cantidad. Sin embargo, la interacción entre ambos factores es muy relevante para la medición de la gravedad específica (Tabla 4, Fig. 2). Se obtienen poros de diámetro horizontal muy grande al utilizar $50 \%$ de almidón de yuca y entre $1.0 \%$ y $1.5 \%$ de emulsificantes y poros muy pequeños con $0 \%$ de ambos (Fig. 1). Si se desea obtener una torta esponjosa y un batido con una gravedad específica menor a 1.0, se deben utilizar emulsificantes entre $1.5 \%$ y $2.0 \%$ y almidón de yuca entre $10 \%$ y $20 \%$ (Fig. 1).

La altura de la torta después del horneo fue una variable principalmente afectada por la cantidad de emulsificantes que contenía la formulación. Los huevos aportaron en gran parte a la emulsión y homogeneización de la mezcla; sin embargo, otros agentes emulsificantes proporcionaron mucha suavidad y esponjosidad al producto final (Tabla 3). La adición de emulsificantes, almidón de yuca y su interacción, fueron factores significativos en la altura de torta. El aumento de almidón de yuca afectó negativamente la altura de la torta ya que esta no creció durante el horneo y sus características sensoriales finales no fueron agradables debido a que presentó dificultad al partir la tajada y la miga fue muy compacta para este tipo de producto. Se observa una relación de almidón de yuca entre 10\% y 20\% y emulsificantes entre $1.0 \%$ y $2.0 \%$ para tortas con alturas superiores a $55 \mathrm{~mm}$ (Fig. 1).
Tabla 3. Análisis de varianza ANOVA para el tamaño de poro, la gravedad específica y la altura en relación al porcentaje de almidón de yuca y emulsificantes en tortas libres de gluten.

\begin{tabular}{|c|c|c|c|c|c|}
\hline & $\begin{array}{l}\text { Suma de } \\
\text { cuadrados }\end{array}$ & $\begin{array}{l}\text { Grados de } \\
\text { libertad }\end{array}$ & $\begin{array}{c}\text { Media } \\
\text { cuadrática }\end{array}$ & $\mathbf{F}$ & Valor $p$ \\
\hline \multicolumn{6}{|l|}{ TAMAÑO DE PORO } \\
\hline Modelo & 0.78 & 5 & 0.16 & 10.73 & 0.0105 \\
\hline A-Emulsificantes (\%) & 0.43 & 1 & 0.43 & 29.18 & 0.0029 \\
\hline B-Almidón de yuca (\%) & 0.44 & 1 & 0.44 & 30.41 & 0.0027 \\
\hline$A B$ & 0.1 & 1 & 0.1 & 7.14 & 0.0442 \\
\hline$A^{2}$ & 0.02 & 1 & 0.02 & 1.37 & 0.2941 \\
\hline$B^{2}$ & 0.008216 & 1 & 0.008216 & 0.56 & 0.4864 \\
\hline Total & 0.85 & 10 & & & \\
\hline \multicolumn{6}{|c|}{ GRAVEDAD ESPECÍFICA } \\
\hline Modelo & 0.053 & 5 & 0.11 & 31.8 & 0.0009 \\
\hline A-Emulsificantes (\%) & 0.037 & 1 & 0.037 & 111.57 & 0.0001 \\
\hline B-Almidón de yuca (\%) & 0.00363 & 1 & 0.00363 & 10.86 & 0.0216 \\
\hline$A B$ & 0.012 & 1 & 0.012 & 37.07 & 0.0017 \\
\hline$A^{2}$ & 0.00231 & 1 & 0.00231 & 6.91 & 0.0466 \\
\hline$B^{2}$ & 0.013 & 1 & 0.013 & 37.88 & 0.0016 \\
\hline Total & 0.055 & 10 & & & \\
\hline \multicolumn{6}{|l|}{ ALTURA } \\
\hline Modelo & 2256.26 & 5 & 451.25 & 35.79 & 0.0006 \\
\hline A-Emulsificantes (\%) & 517.54 & 1 & 517.54 & 41.05 & 0.0014 \\
\hline B-Almidón de yuca (\%) & 718.18 & 1 & 718.18 & 56.96 & 0.0006 \\
\hline$A B$ & 260.44 & 1 & 260.44 & 20.66 & 0.0061 \\
\hline$A^{2}$ & 40.17 & 1 & 40.17 & 3.19 & 0.1343 \\
\hline$B^{2}$ & 431.69 & 1 & 431.69 & 34.24 & 0.0021 \\
\hline
\end{tabular}

23193 
Figura 1. Superficie de respuesta para el tamaño de poro, gravedad específica y altura de la torta en relación al porcentaje de almidón de yuca y emulsificantes en tortas libres de gluten.
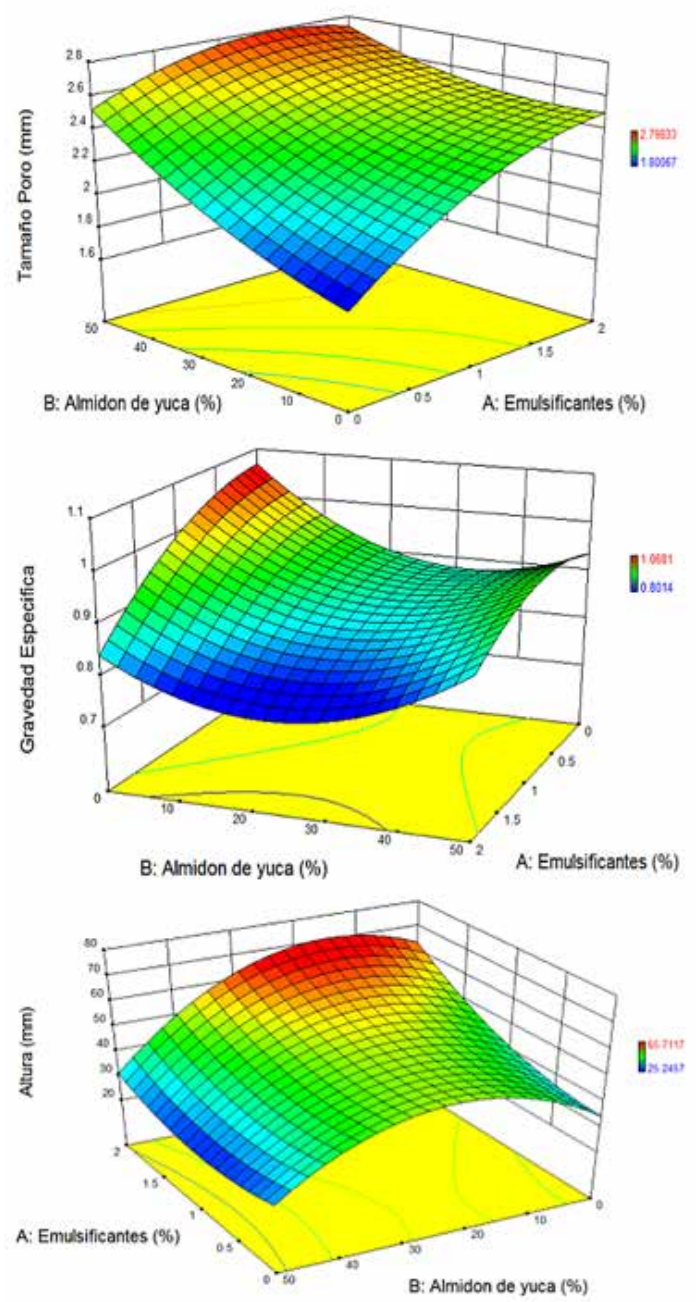

Los modelos que explican cada una de las variables evaluadas y su variación de acuerdo a la cantidad de emulsificantes y almidón de yuca usada son:

$$
\text { Altura }=53.22+8.21 \mathrm{~A}-9.84 \mathrm{~B}-6.34 \mathrm{AB}
$$

Gravedad especifica $=0.87-0.070 \mathrm{~A}-0.022 \mathrm{~B}+0.044 \mathrm{AB} \quad$ (4)

Tamaño de poro $=2.44+0.24 \mathrm{~A}+0.24 \mathrm{~B}-0.13 \mathrm{AB}$

Donde A: \%Emulsificantes y B: \%Almidón de yuca.
Las variables respuesta fueron optimizadas así: Emulsificantes (0\%-1,5\%). Almidón de yuca (0\%-20\%), gravedad específica minimizando (0.8014), tamaño de poro (1,9-2,5mm), altura maximizando $(65,711 \mathrm{~mm})$. A partir de estos parámetros establecidos, el programa arrojó tres soluciones y se utilizó los siguientes parámetros del punto óptimo para realizar nuevamente la torta: Emulsificantes 1,5\%, almidón de yuca 19,64\%, gravedad específica 0,829 , tamaño de poro $2,485 \mathrm{~mm}$, y altura $60,774 \mathrm{~mm}$. Se realizaron las mismas mediciones de los once experimentos iniciales (Tabla 4).

Tabla 4. Tamaño de poro, altura de la torta y gravedad espećífica; realizadas a una torta libre de gluten con los parámetros del punto óptimo.

\begin{tabular}{c|ccc|ccc|ccc|} 
Replicas & \multicolumn{2}{|c|}{$\begin{array}{c}\text { Tamaño del poro } \\
(\mathbf{m m})\end{array}$} & \multicolumn{3}{c|}{$\begin{array}{c}\text { Altura de la torta } \\
(\mathbf{m m})\end{array}$} & \multicolumn{3}{|c|}{ Gravedad especifica } \\
\hline $\mathbf{1}$ & 2,25 & 2,25 & 2,26 & 60,08 & 60,07 & 60,08 & 0,893 & 0,891 & 0,891 \\
$\mathbf{2}$ & 2,48 & 2,48 & 2,49 & 59,98 & 60,01 & 59,79 & 0,887 & 0,89 & 0,889 \\
$\mathbf{3}$ & 2,26 & 2,26 & 2,27 & 60,01 & 60,04 & 60,04 & 0,891 & 0,895 & 0,892 \\
\hline
\end{tabular}

Con el fin de comparar los datos teóricos con los experimentales, y comprobar si los modelos de cálculo obtenidos para cada variable las explican en un gran porcentaje, se realizó una comparación entre el modelo teórico y experimental (Fig. 2). Hubo una coincidencia clara entre las medias del valor experimental y el modelo teórico para el tamaño de poro y la altura de la torta. Sin embargo, no se vio una evidente relación entre los valores de la gravedad específica, aunque, se pudo observar que la variación es muy pequeña y tomó valores entre 0.82 y 0.90 , lo cual no refleja una diferencia significativa en los resultados.

Figura 2. Comparación de medias en el Test de Fisher del modelo experimental y teórico para tamaño de poro, atura de la torta y gravedad espećífica.

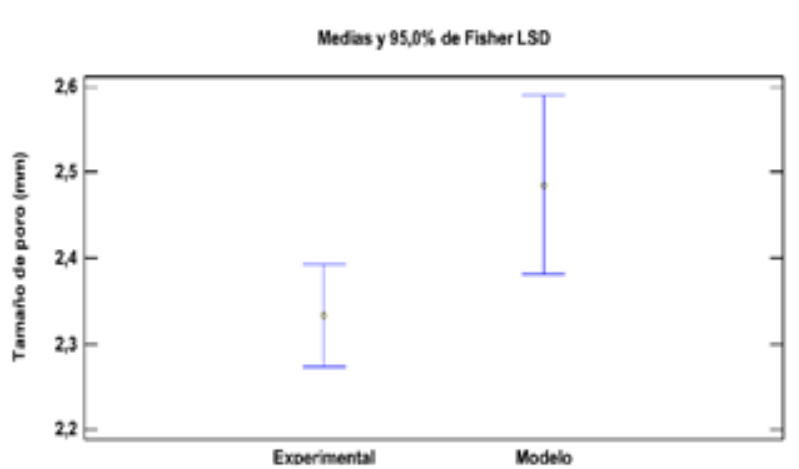


1 a
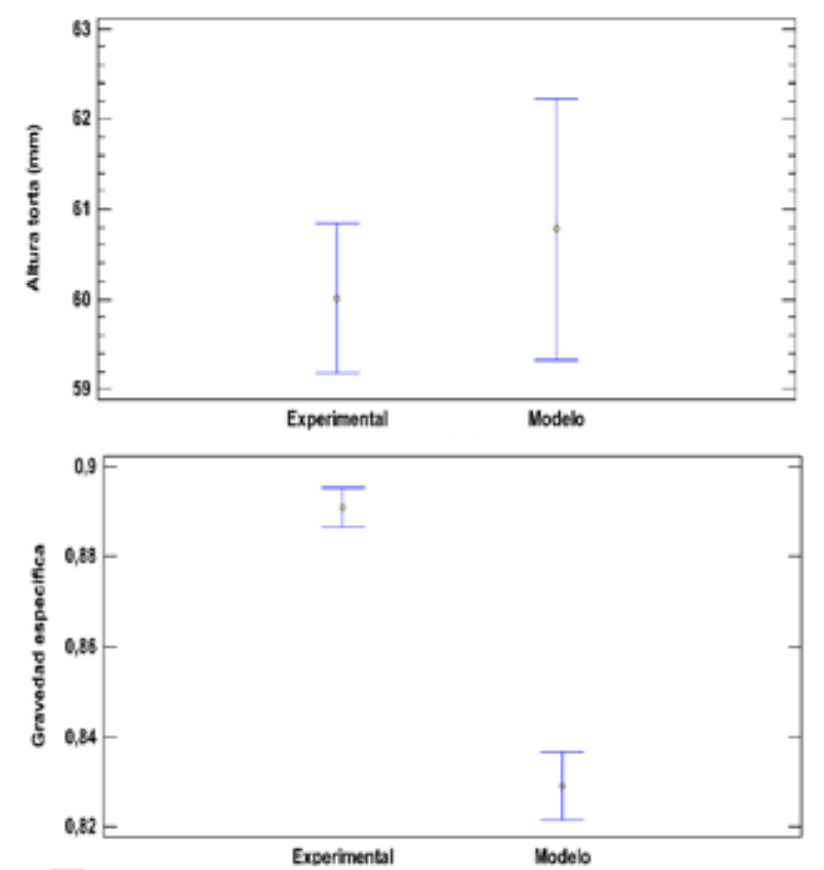

\section{DISCUSIÓN}

El arroz y el maíz están entre los cereales considerados aptos para ser consumidos por la población celíaca y ha sido estudiados como sustituto del trigo en la formulación de productos panificados [17]. Sin embargo, los productos elaborados con arroz o maíz como sustitutos del trigo raramente cuentan con buena aceptabilidad, ya que no tienen la propiedad de formar una fase continua y una buena estructura de masa cuando son mezcladas con el agua. Por esta razón, se incorpora emulsificantes a la formulación ingredientes capaces de aportar propiedades viscoelásticas, aspecto de fundamental importancia para la formación y el establecimiento de la estructura que determina la textura final de los productos elaborados con harina de trigo [18].

Los almidones nativos de diferentes especies de vegetales tienen como característica fundamental que sus propiedades fisicoquímicas y funcionales estarán influenciadas por sus estructuras granulares y moleculares. Las propiedades más importantes a considerar para determinar la utilización del almidón en la elaboración de alimentos y otras aplicaciones industriales incluyen las fisicoquímicas: gelatinización y retrogradación; y las funcionales: solubilidad, hinchamiento, absorción de agua, sinéresis y comportamiento reológico de sus pastas y geles [19,20]. Estudios sugieren que el uso del almidón de yuca, con la adición de margarina y clara de huevo, reducen la magnitud de gelatinización y solubilización del almidón en productos panificables, aumentando la cantidad de aire atrapado durante el batido de la masa en la fase de mezclado [21]
En este estudio se determinó las proporciones óptimas para la elaboración de una torta libre de gluten que cumpla unas características de textura específicas, usando como mejoradores almidón de yuca y estabilizantes. Se evidenció que altas cantidades de almidón de yuca producen características indeseadas en el producto como apelmazamiento, tamaño de poro más altos y sensaciones granulosas a nivel sensorial. La adición de la mezcla de emulsificantes influyó positivamente durante su evaluación y en todas las cantidades analizadas; ya que son moléculas con un extremo afín a los líquidos y hacen posible la formación de una emulsión estable, homogénea y fluida. Propiedades como tamaño de poro, altura obtenida y gravedad específica son parámetros de gran funcionalidad al evaluar las características texturales de un producto panificable; ya que permiten controlar e identificar las irregularidades dentro del alimento; así como determinar sensaciones y cualidades sensoriales. Es necesario, sin embargo, tener en cuenta que dentro de la elaboración del producto se encuentran factores importantes que inciden directamente en la elaboración de la torta como son el orden de adición de los ingredientes, el tiempo de batido, el tipo de horno, la temperatura y el tiempo de horneo.

El diseño experimental demostró diferencias significativas al adicionar las diferentes cantidades de emulsificantes a la torta, ya que los mono- y diglicéridos de los ácidos grasos favorecen la incorporación de aire en la masa durante el batido, mejorando el volumen y la textura. Estos factores se vieron reflejados en las cantidades necesarias de mejoradores para obtener una torta con propiedades de textura óptimas y similares a las aportadas por la harina de trigo, usando sustitutos libres de gluten (harina de arroz y de maíz). De igual modo se observaron en las diferentes aplicaciones de los mejoradores para el batido, que las características texturales se afectaron directamente, encontrando así diferencias entre los tamaños de poro, altura de la torta y propiedades sensoriales que variaba según la proporción seleccionada.

La aceptación de los consumidores por aspectos visuales, de sabor y textura juega un papel importante en la producción panadera [22]. La textura es un indicador importante de frescura y calidad para el consumidor, siendo un factor clave para la aceptación del producto en el mercado [23]. Los productos elaborados con otro tipo de harinas a menudo poseen menor volumen y migas y cortezas más grandes [24]. La inclusión de almidón de yuca y emulsificantes en proporciones adecuadas afecta positivamente las características sensoriales de los productos obtenidos [24].

\section{AGRADECIMIENTOS}

Agradecemos a la asesora Luz Mery Echeverri, Profesor Esteban Largo y Óscar Vega, al Ingeniero David Velásquez y a los monitores del Laboratorio de Materiales de la Facultad de Ingeniería de la Universidad de Antioquia. 
[1] Biesiekierski, J R. (2017). What is gluten? Journal of Gastroenterology and Hepatology, 32(S1), 78-81. doi: http://doi. Biesiekierski, J.R. (2017).
org/10.1111/jgh. 13703

[2] de Souza, M. C. P., Deschênes, M. E., Laurencelle, S., Godet, P., Roy, C. C., \& Djillali-Saiah, I. (2016). Pure oats as part of the Canadian gluten-free diet in celiac disease: The need to revisit the issue. Canadian Journal of Gastroenterology and Hepatology, 2016(1576360), 1-8. doi: http://dx.doi.org/10.1155/2016/1576360

[3] Comino, I., Moreno, M. L., \& Sousa, C. (2015). Role of oats in celiac disease. World Journal of Gastroenterology, 21(41) 11825-11831. doi: https://dx.doi.org/10.3748\%2Fwjg.v21.441.11825

[4] Lebwohl, B., Eriksson, H., Hansson, J., Green, P. H. R., \& Ludvigsson, J. F. (2014). Risk of cutaneous malignant melanoma in patients with celiac disease: A population-based study. Journal of the American Academy of Dermatology, 71(2), 245-248. doi: https://doi.org/10.1016/j.jaad.2014.03.029

[5] FDA (2016) Gluten-Free Labeling of Foods. U.S. Food \& Drug Administration. Recuperado de https://www.fda.gov/food/ guidanceregulation/guidancedocumentsregulatoryinformation/allergens/ucm 362510 .htm

[6] Singh, J., \& Whelan, K. (2011). Limited availability and higher cost of gluten-free foods. Journal of Human Nutrition and Dietetics, 24(5), 479-486. doi: http://doi.org/10.1111//.1365-277X.2011.01160.x

[7] Whitaker, J. K. H., West, J., Holmes, G. K. T., \& Logan, R. F. A. (2009). Patient perceptions of the burden of coeliac disease and its treatment in the UK. Alimentary Pharmacology and Therapeutics, 29(10), 1131-1136. doi: http://doi. org/10.1111/j.1365-2036.2009.03983.x

[8] Hager, A. S., \& Arendt, E. K. (2013). Influence of hydroxypropylmethylcellulose (HPMC), xanthan gum and their combination on loaf specific volume, crumb hardness and crumb grain characteristics of gluten-free breads based on rice, maize, teff and buckwheat. Food Hydrocolloids, 32(1), 195-203. doi: https://doi.org/10.1016/j based on rice, maize,
foodhyd.2012.12.021

[9] Premlal Ranijth, H.M. \&Wijewardene, U. (2006) Lipid emulsifiers and surfactants in dairy and bakery products. En F.D. Gunstone (Ed.), Modifying Lipids for Use in Food (pp. 393-428).

[10]Ranjith, H. P., \& Wijewardene, U. (2006). Lipid emulsifiers and surfactants in dairy and bakery products. In Modifying Lipids for Use in Food (pp. 393-428).Woodhead Publishing Series in Food Science, Technology and Nutrition, Boca Raton: Woodhead Publishing. doi: https:///doi.org/10.1533/9781845691684.3.393

[11] Rodríguez Garcia, J. (2014) Reformulacion de productos horneados para disminuirel contenido en grasay azucar mediante la sustitucion con inulina: Efectos sobre la estructura y propiedades fisicas. (Tesis Doctoral). Universitat Politecnica de Valencia, Valencia.

[12] Morales Moreno, M.M. (2013). Evaluacion de la adicion de emulsificantes y componentes fisiologicamente activos en la elaboracion de un buñuelo (Tesis de Maestría). Universidad Nacional de Colombia, Medellin.

[13] Saeleaw, M., \& Schleining, G. (2010). Effect of blending cassava starch, rice, waxy rice and wheat flour on physicohemical properties of flour mixtures and mechanical and sound emission properties of cassava crackers Jounnal of Food Engineering, 100(1), 12-24.doi: https://doi.org/10.1016/j.jfoodeng.2010.03.020

[14]. Conforti, F.D. (2007) Cake Manufacture. En Y.H. Hui (Ed.), Bakery Products:Science and Technology (pp. 393-410). Amez, IA: Blackwell Publishing.

[15] Hesso, N., Garnier, C., Loisel, C., Chevallier, S., Bouchet, B. \& Le-Bail A. (2015). Formulation effect study on batter and cake microstructure: Correlation with rheology and texture. Food Structure, 5, 31-41. doi: https:///doi. org/10.1016/1 foostr.2015.03.002

[16] Rosenthal, A.J. (2001) Textura de los alimentos. Zaragoza: Editorial Acribia.

[17] 0'Donnell, K. (2016) Manual de Produccion de Panaderia. Xlibris.

[18] Gujral, H. S., \& Rosell, C. M. (2004). Improvement of the breadmaking quality of rice flour by glucose oxidase. Food Research International, 37(1), 75-81. doi: https://doi.org/10.1016/j.foodres.2003.08.00

[19] Clerici Pedrosa, S., \& El-Dash, M.T. (2006) Harina extrusada de arroz como sustituto de gluten en produccion de pan. Archivos Latinoamericanos de Nutrición, 56.
[20] Wang, L. Z., \& White, P.J. (1994). Structure and properties of amylose, amylopectin, and intermediate materials of oat starches. Cereal Chemistry, 71(3), 263-268.

[21] Hernández-Medina, Marilyn, Torruco-Uco, Juan Gabriel, Chel-Guerrero, Luis, \& Betancur-Ancona, David. (2008). Caracterizacín fisicoquímica de almidones de tubérculos cultivados en Yucatán México Food Science and Technology (Campinas), 28(3), 718-726. https://dx.doi. org/10.1590/50101-20612008000300031

[22] Eggleston, G. (1992). Can we make a marketable cassava bread without wheat? Cassava Newsletter (CIAT), 16(1), 7-8. [23] Siro, L. Kápolna, E., Kápolna, B. \& L Lugasi, A. (2008). Functional food. Product development, marketing and consumer acceptance一A review. Appetite, 51(3), 456-467. doi: https://doi. org/10.1016/.jappet.2008.05.060

[24] Brady, P. L., \& Mayer, S. M. (1985). Correlation of sensory and instrumental measures of bread texture. Cereal Chemistry, $62(1), 70-72$.

[25] Heiniö, R. L. (2006). Sensory attributes of bakery products. Bakery products: science and technology, 285-298.

\section{CONTRIBUCIÓN DE LOS AUTORES}

Mariana Vélez Gutierrez, Laura Cristina Jimenez y Ximena Yepes Sánchez trabajaron en la concepción del estudio, desarrollo de los experimentos, análisis de los datos y redacción del manuscrito. 